\title{
An overview of AVIRIS-NG airborne hyperspectral science campaign over India
}

\author{
Bimal K. Bhattacharya ${ }^{1, *}$, Robert O. Green ${ }^{2}$, Sadasiva $\operatorname{Rao}^{3}$, M. Saxena ${ }^{1}$, \\ Shweta Sharma ${ }^{1}$, K. Ajay Kumar ${ }^{1}$, P. Srinivasulu ${ }^{3}$, Shashikant Sharma ${ }^{1}$, \\ D. Dhar ${ }^{1}$, S. Bandyopadhyay ${ }^{4}$, Shantanu Bhatwadekar ${ }^{4}$ and Raj Kumar ${ }^{1}$ \\ ${ }^{1}$ Space Applications Centre, Indian Space Research Organisation, Ahmedabad 380 015, India \\ ${ }^{2}$ Jet Propulsion Laboratory, California Institute of Technology, CA 91109, USA \\ ${ }^{3}$ National Remote Sensing Centre, Indian Space Research Organisation, Hyderabad 500 625, India \\ ${ }^{4}$ Earth Observation Science Directorate, Indian Space Research Organisation, Bengaluru 560 231, India
}

\begin{abstract}
The first phase of an airborne science campaign has been carried out with the Airborne Visible/Infrared Imaging Spectrometer Next Generation (AVIRIS-NG) imaging spectrometer over 22,840 sq. $\mathrm{km}$ across 57 sites in India during 84 days from 16 December 2015 to 6 March 2016. This campaign was organized under the Indian Space Research Organisation (ISRO) and National Aeronautics and Space Administration (NASA) joint initiative for HYperSpectral Imaging (HYSI) programme. To support the campaign, synchronous field campaigns and ground measurements were also carried out over these sites spanning themes related to crop, soil, forest, geology, coastal, ocean, river water, snow, urban, etc. AVIRIS-NG measures the spectral range from 380 to $2510 \mathrm{~nm}$ at $5 \mathrm{~nm}$ sampling with a ground sampling distance ranging from 4 to $8 \mathrm{~m}$ and flight altitude of 4-8 km. On-board and ground-based calibration and processing were carried out to generate level 0 (LO) and level 1 (L1) products respectively. An atmospheric correction scheme has been developed to convert the measured radiances to surface reflectance (level 2). These spectroscopic signatures are intended to discriminate surface types and retrieve physical and compositional parameters for the study of terrestrial, aquatic and atmospheric properties. The results from this campaign will support a range of objectives, including demonstration of advanced applications for societal benefits, validation of models/techniques, development of state-of-the-art spectral libraries, testing and refinement of automated tools for users, and definition of requirements for future space-based missions that can provide this class of measurements routinely for a range of important applications.
\end{abstract}

Keywords: Airborne science campaign, hyperspectral sensing, imaging spectrometer, surface reflectance.

\section{Introduction}

HYPERSPECTRAL sensing (HSS) of the land, water and atmosphere is based on the technique of imaging spec-

\footnotetext{
*For correspondence. (e-mail: bkbhattacharya@sac.isro.gov.in)
}

troscopy, where a complete spectrum is measured for every point in an image. These spectra record the interaction of energy with matter and enable the use of advanced spectroscopic methods to determine the properties of the materials measured. Molecular and structural or particle characteristics of the surface or atmosphere produce diagnostic spectral features in the measurements. These features arise from electronic transition and vibrational processes as well as scattering effects, and are related to the fundamental material composition. Generally, electron transition signatures occur at shorter wavelengths related to changes in energy state of electrons bound to atoms, molecules or lattices. Vibrational signatures typically occur at longer wavelengths due to molecular bond stretching and bending along with related overtones.

HSS refers to the measurement of 100s of contiguously sampled spectral channels with a relatively narrow sampling interval of $1-15 \mathrm{~nm}$. In contrast, Multi-Spectral Sensing (MSS) typically corresponds to 5-10 discrete bands with bandwidths of 50-400 nm.

Advantages of HSS are: (i) detection of more materials or surface types such as minerals, rocks, vegetation, snow; (ii) capture of full spectral signatures related directly to composition; (iii) recording the absorption strength that can be related to the abundance of the materials present, and (iv) the ability to derive sub-pixel abundance estimates for multiple materials recorded in a spectrum. HSS offers opportunities for new science and applications with broad societal benefits. The technology-enabling development of high-fidelity HSS instruments has only recently begun (Airborne Visible/Infrared Imaging Spectrometer Next Generation (AVIRIS-NG) was developed in 2012). Laboratory and portable point spectrometers have been available for a longer period, but are severely limited in their sampling ability. Recently, several new HSS instruments such as micro-hyperspec and nanohyperspec have become available providing centimetre spatial resolution from airborne, vehicle and mastmounted platforms. Technology demonstration through HSS spaceborne missions, such as EO-1 Hyperion of National Aeronautics and Space Administration (NASA), 
USA, provided sample datasets over the world, including India, but suffered from low signal-to-noise ratio (SNR) and limited data availability. However, the potential applicability of HSS over India was tested with Hyperion for agriculture (e,g. mustard crop disease $)^{1}$, and geology (e.g. Dongargarh, Rajasthan $)^{2}$. In addition, several ground-based studies in India using ground-based spectrometers demonstrated the advantages of using HSS data for crops, soils, geology, etc. ${ }^{3-5}$. The recent hyperspectral science initiative also includes Network Programme on Imaging Spectroscopy and Applications (NISA) ${ }^{6}$ of Department of Science and Technology (DST), Government of India (GoI).

To examine the utility of HSS, Indian Space Research Organisation (ISRO) has developed and flown several hyperspectral instruments. Hyperspectral Imager (HySI) aboard Indian Mini Satellite (IMS-1), launched on 28 April 2008, was India's first space-based imaging spectrometer for oceanic and atmospheric sciences. Chandrayaan-1, India's first mission to the Moon, launched on 22 October 2008, carried HySI payload which provided spectra of lunar surface in the spectral range 421-964 nm in 64 contiguous bands. Apart from space-based missions, several airborne surveys have been carried out by ISRO using hyperspectral sensors. For example, Space Applications Centre (SAC), ISRO developed and flew the Airborne Imaging Spectrometer (AIMS) in 1996 with an SNR $>400$ and operating wavelength range 454-888 nm; Airborne Hyperspectral Imager (AHySI; 2007) having SNR $>100$ and operating wavelength range 465-995 nm; Airborne Imaging Spectrometer-2 (AIMS-2; 2014); Airborne Imaging Spectrometer-3 (AIMS-3; 2017). AIMS was the first spectrometer developed by SAC, ISRO in 1996. It had 143 bands covering the spectral range from 454 to $888 \mathrm{~nm}$ with spectral resolution from 2.8 to $4.3 \mathrm{~nm}$ and SNR of about 400 . The instrument utilized commercial optical system for the fore optics, collimating and focusing optics ${ }^{7}$. The dispersion was obtained with a plane grating. AHySI was developed by SAC, ISRO in 2007. It is based on the wedge filter and covers the spectral range from 465 to $995 \mathrm{~nm}$ in 512 contiguous bands with spectral resolution of $15 \mathrm{~nm}$ and SNR $>100$.

Looking ahead with international collaboration opportunities, the ISRO and NASA joint airborne hyperspectral (HySI) science campaign over India was conceptualized. This campaign selected AVIRIS-NG developed by Jet Propulsion Laboratory (NASA) in 2012. AVIRIS-NG is the next generation of AVIRIS $^{8}$ and also uses technologies of the Moon Mineralogy Mapper (M3) ${ }^{9}$ flown as a guest instrument on Chandrayaan-1. AVIRIS-NG uses state-of-theart technology to deliver high-fidelity spectroscopic measurements. For example, the convex grating of AVIRISNG enables high SNR as well as high spatial and spectral uniformity, thus nearly eliminating artefacts such as 'smile' and 'keystone' that can be present in other hyperspectral instruments. AVIRIS-NG supports the objective of exploring and developing new spectroscopic techniques and tools for a variety of scientific investigations that are relevant to the diverse natural and anthropogenic environment of India. AVIRIS-NG campaign flights were conducted during the period from December 2015 to March 2016 (as Phase 1) with the following broad objectives under the ISRO and NASA HySI programme:

- To harness the benefits of unique and advanced remote sensing measurements for society by bringing together important talents and expertise in instrumentation, science, and applications of both ISRO and NASA.

- To jointly develop advanced scientific understanding, models, algorithms and techniques through knowledge sharing and to open up new avenues in this regard.

- To provide the required precursor ground-truth data, research demonstrations related to the utility of future space-borne imaging spectrometer missions.

\section{Study sites and AVIRIS-NG instrument}

AVIRIS-NG was deployed for the ISRO and NASA airborne campaign on board an ISRO B200 aircraft with nadir view port. AVIRIS-NG measures the spectral range $380-2510 \mathrm{~nm}$ at $5 \mathrm{~nm}$ intervals with high SNR (>2000@ $600 \mathrm{~nm}$ and >1000@2200 nm) and an accuracy of 95\%. The field-of-view (FOV) is $34^{\circ}$ and instantaneous FOV (IFOV) is 1 milliradian. Ground sampling distance (GSD) varies from 4 to $8 \mathrm{~m}$ for flight altitude of $4-8 \mathrm{~km}$ with swaths ranging from 3 to $6 \mathrm{~km}$. AVIRIS-NG includes an INS/GPS system that enables full reconstruction of the airborne measurements to an ortho-rectified product. The science themes of this campaign are spread over 8 broad areas with a total 57 sites (Table 1) based on proposals by scientists from various ISRO centres, universities and Indian Institute of Technologies (IITs).

Figures 1 and 2 show the geographical distribution of sites, and some examples of their false colour composites (FCCs) from Indian Remote Sensing (IRS) satellite sensors with flight paths respectively.

\section{Campaign coordination, execution and ground-truth data collection}

After the pre-campaign activities, including the crosscalibration of different sets of field instruments, an exhaustive field campaign started from 19 December 2015 and continued till 8 March 2016. Before starting the science campaign flights, calibration and validation activities were carried out to evaluate the performance of AVIRIS-NG with respect to the ground measured spectra. The flight campaign was organized from nine airport bases (Table 2)-Ahmedabad, Udaipur, Bhubaneswar, Hyderabad, Mangaluru, Coimbatore, Chandigarh, Patna 
Table 1. Science themes and number of study sites in each theme during Phase 1 campaign

\begin{tabular}{lcc}
\hline Broad science themes & Sub-themes & Number of study sites \\
\hline Agriculture and ecosystem & Crop, soil, mangrove, wetland & 21 \\
Geology & Coastal zone, biological oceanography, coral reef & 11 \\
Ocean and coastal & & 11 \\
Rivers and water quality & & 5 \\
Urban & \multirow{2}{*}{ Air quality, cloud microphysics } & 2 \\
Snow and ice & & 2 \\
Atmosphere & & 3 \\
Calibration and validation & & 2 \\
\hline
\end{tabular}

Table 2. AVIRIS-NG airborne hyperspectral Phase-1 campaign diary

\begin{tabular}{llcc}
\hline $\begin{array}{l}\text { Airports (in chronological order } \\
\text { according to flight schedule) }\end{array}$ & \multicolumn{1}{c}{ Duration } & $\begin{array}{c}\text { Number } \\
\text { of sites }\end{array}$ & $\begin{array}{c}\text { Area imaged } \\
\text { (sq. km) }\end{array}$ \\
\hline Begumpet & $\begin{array}{l}\text { 16-21 December 2015 } \\
\text { 25-29 January 2016 }\end{array}$ & 12 & 2650 \\
Bhubaneswar & 22-28 December 2015 & 6 & 3780 \\
Mangaluru & 29 December 2015-2 January 2016 & 5 & 3491 \\
Coimbatore & 3-8 January 2016 & 5 & 1416 \\
Udaipur & 31 January-5 February 2016 & 8 & 3697 \\
Ahmedabad & 6-16 February 2016 & 10 & 2788 \\
Chandigarh & 6-9 March 2016 & & \\
Patna & 17-21 February 2016 & 4 & 835 \\
Kolkata & 22-24 February 2016 & 3 & 396 \\
\hline
\end{tabular}

Total number of days $=84$; total area imaged $=22,840$ sq. $\mathrm{km}$ over 57 sites.

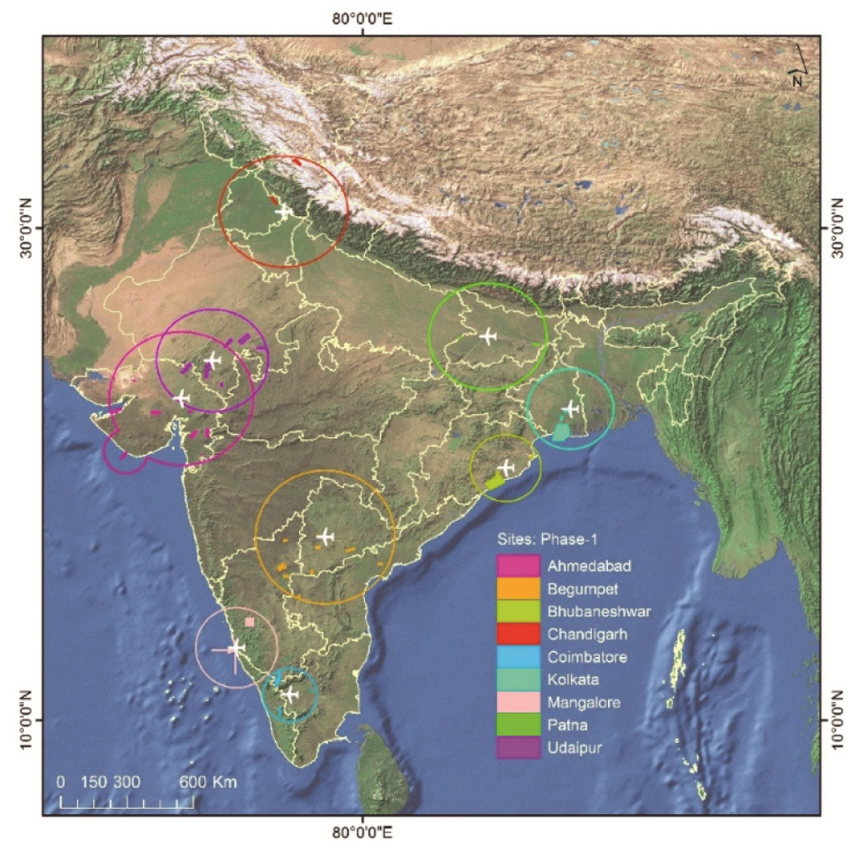

Figure 1. Geographical distribution of AVIRIS-NG science campaign Phase 1 sites.

and Kolkata. More than 200 people participated in the field campaigns. Amalgamation of different communication techniques helped in on-site coordination for mobility of field teams to adjust to last minute changes in flight schedule depending upon Air Traffic Control (ATC) clearances, weather and other factors. The high-resolution short-range weather forecast on cloud fraction from MOSDAC (Meteorological and Oceanic Satellite Data Archival Centre; mosdac.gov.in) was provided to the campaign coordination teams and field campaign teams for effective and efficient flight planning and field data collection.

A large number of ground measured field spectra and associated field data have been collected coincident with the airborne campaign dates. Figure 3 provides examples of AVIRIS-NG spectral signatures over different targets. Apart from the supporting ground spectroscopic measurements, IRS Resourcesat-2, LISS IV and RISAT-1 FRS-1 data were collected at near coincidence to the airborne campaign flights for highly dynamic sites (e.g. agriculture).

Laboratory-based chemical analyses was carried out on soil, plant, rock and water samples collected during field campaigns. These in situ data and analyses have been used along with AVIRIS-NG data to demonstrate the utility of imaging spectroscopy measurements, to develop new algorithms, tools, techniques, enhanced science understanding and refine requirements for future spacebased satellite observations.

In order to develop the human resources among different research agencies and academia, and to develop the 


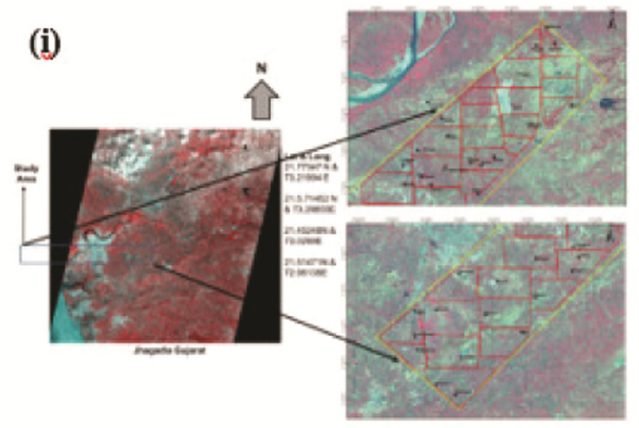

(ii)

(iv)

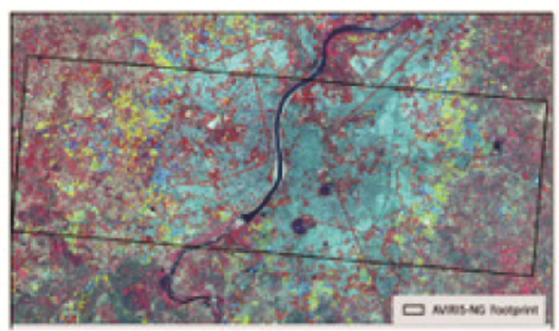

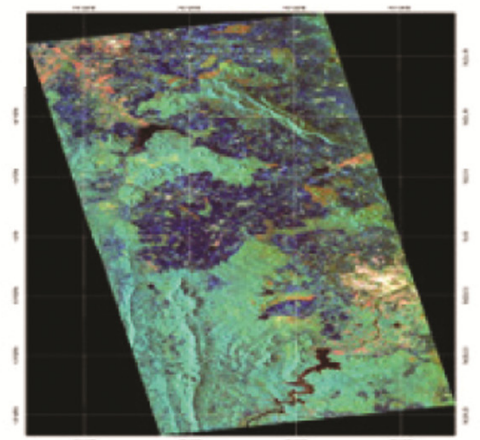

(iii)

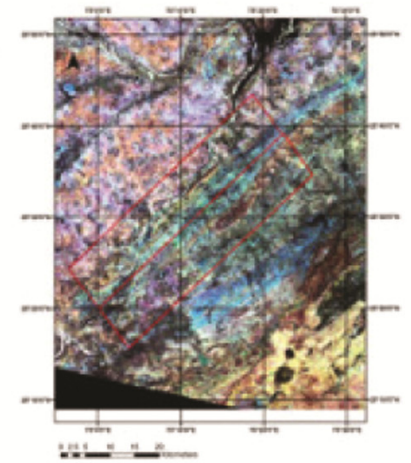

(v)

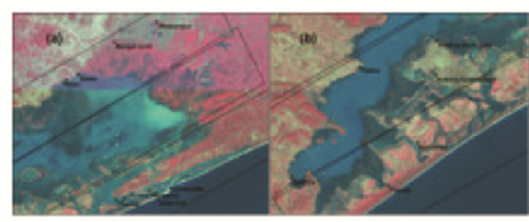

(vi)

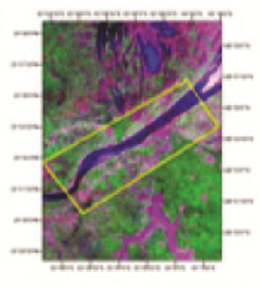

Figure 2. Examples of sites and flight paths for (i) agriculture, (ii) forest, (iii) geology, (iv) urban, (v) coastal-ocean and (vi) river.
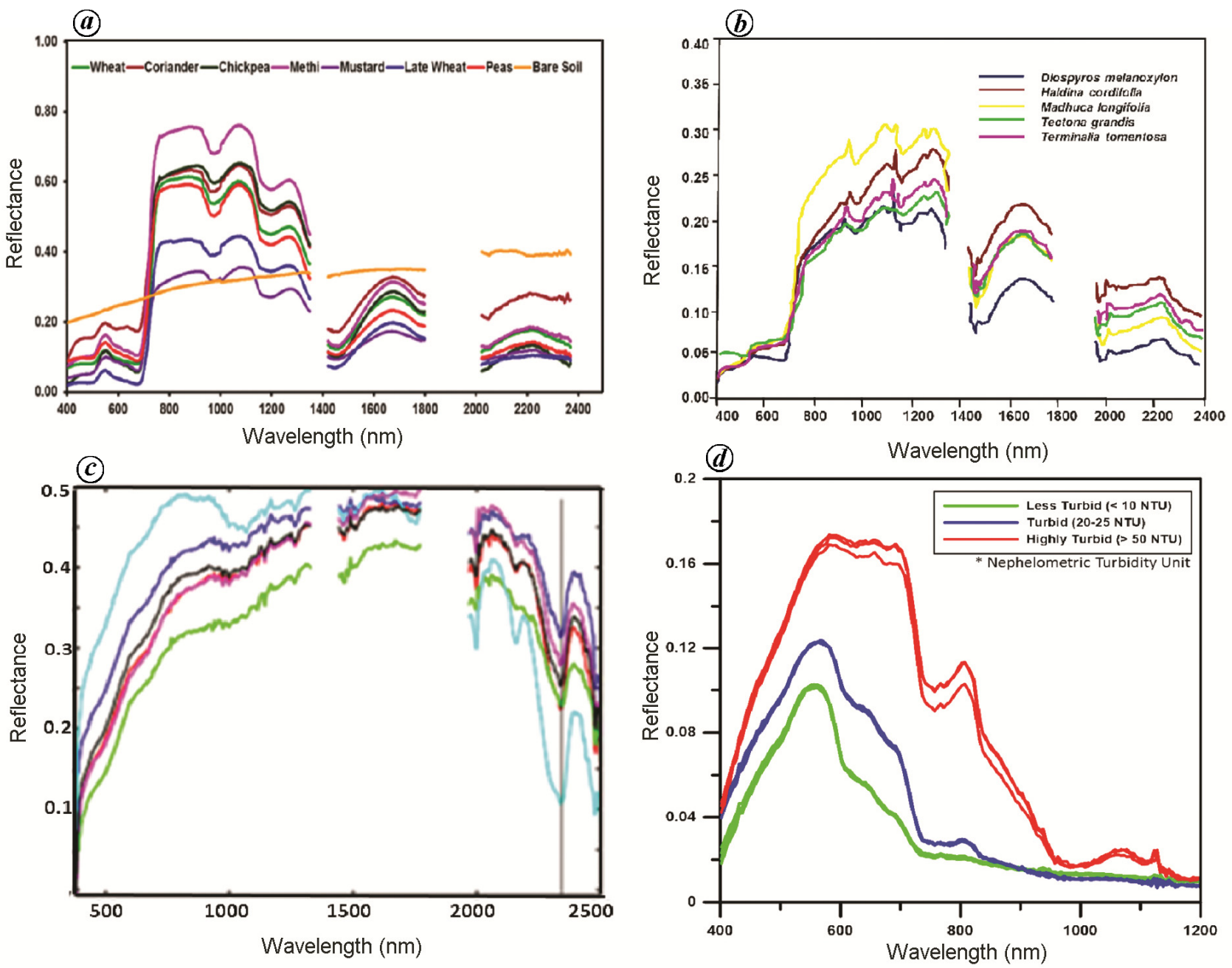

Figure 3. Examples of typical hyperspectral signatures derived from AVIRIS-NG over different surface types such as $(\boldsymbol{a})$ crop and soil, $(\boldsymbol{b})$ forest, $(\boldsymbol{c})$ minerals and $(\boldsymbol{d})$ water. 
Table 3. Product type, definition and correction levels

\begin{tabular}{lll}
\hline $\begin{array}{l}\text { Data product } \\
\text { levels }\end{array}$ & \multicolumn{1}{c}{ Product types } & \multicolumn{1}{c}{ Product content } \\
\hline Level-0 & Raw & $\begin{array}{l}\text { Raw data as captured by the sensor + GLT, IGM } \\
\text { and LOC files } \\
\text { Level-1 }\end{array}$ \\
$\begin{array}{l}\text { Revel-2 } \\
\text { Calibrated radiance } \\
\text { Atmospherically corrected surface } \\
\text { reflectance }\end{array}$ & $\begin{array}{l}\text { Surface reflectance image cube + GLT, } \\
\text { IGM and LOC files }\end{array}$ \\
\hline
\end{tabular}

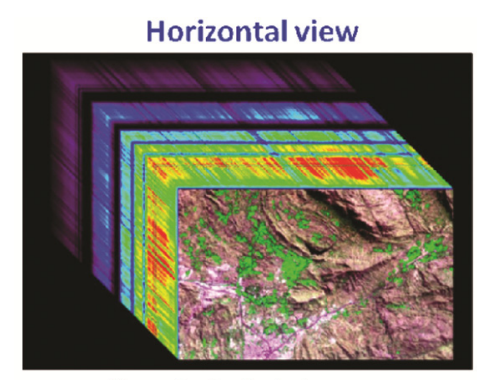

Near Ambaji, Gujarat

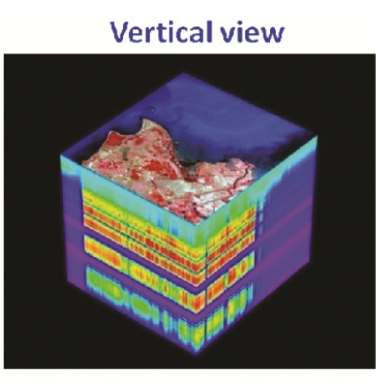

Mangalore coast, Karnataka
Figure 4. Examples of hyperspectral 3D data cubes derived from AVIRIS-NG over different thematic targets.

expertise on the use of this type of spectral imaging data, announcement of opportunity (AO) proposals were invited from different Indian and US research agencies by both ISRO and NASA respectively. The projects to use these new measurements have been selected on a range of factors, including technical merit and innovation.

\section{AVIRIS-NG data products and dissemination}

AVIRIS-NG measurements are represented as 3D data cubes with a spectrum recorded for each sample in an image. Figure 4 shows examples of image cubes while Figure 5 shows examples of surface view as natural colour composites (NCC).

For all sites of the campaign, three levels of AVIRISNG data products (Table 3) were generated: L0, L1 and L2. The L0 are raw data uncompensated for instrument effects. The L1 is the ortho-rectified and calibrated atsensor radiance for the flight altitude of the aircraft. The L0 and L1 products were generated real-time in the AVIRIS-NG instrument on the ISRO B200 aircraft. These products were retained by ISRO at the end of each flight for verification of the sites imaged. Once released, the L2 surface reflectance products were generated using advanced atmospheric correction algorithms. Two types of prototype atmospheric correction models have been developed - point-based and pixel-based. The former is used for science where the atmosphere is assumed spatially invariant and point-measured aerosol optical depth and water vapour can be used for atmospheric correction. The more advanced pixel-based approach estimates atmospheric water vapour and aerosol optical depth pixelby-pixel from AVIRIS-NG radiance itself. These derived atmospheric parameters then serve as inputs to atmospheric correction algorithms. All AVIRIS-NG measurements from this campaign are available as L1 and L2 products.

The AVIRIS-NG data have been archived at the VEDAS geo-portal (https://vedas.sac.gov.in/aviris) of SAC, ISRO) after obtaining due clearances of collected data from Government of India and further processing by JPL (NASA) and SAC (ISRO). Figure 6 shows the different facilities of VEDAS portal and data-flow mechanism. About 230 AVIRIS-NG scenes with data volume of 1.5 TB collected over 57 sites in the first phase of the campaign have been archived. In addition, the associated ground-truth data and field campaign reports are archived in VEDAS. The AVIRIS-NG data have been shared with all Indian announcement of opportunity (AO) PIs as well as other researchers through VEDAS, in accordance with specified guidelines.

\section{Outcome and conclusions}

The major scientific achievements from Phase-1 campaign data include: (i) identification of different sensitive band regions for improved discrimination of crop types, forest species, disease detection, canopy and soil water stresses, soil types, land uses, coral reef macro-algae, minerals, coastal vegetation, urban roof types; (ii) development of retrieval schemes for the determination of crop chlorophylls, ocean chlorophyll, colour dissolved organic matter (CDOM) concentration, suspended sediments and coastal bathymetry, water turbidity parameters and their validation, development of retrieval schemes of different atmospheric parameters such as aerosol optical depth, water vapour, cloud-microphysical parameters and their validation and (iii) snow-grain size characterization and lichen presence verification in alpine areas of the Western Himalayan region with AVIRIS-NG spectra.

After the analysis with Phase-1 data, it was found that another cycle of campaign was required to cover more sites in India and to develop sound India-specific spectral libraries, develop algorithms, models, techniques, tools, calibration and validation, and skilled human resources capable of handling 3D data cubes. This Phase-2 campaign could also capture differing phenology, including 

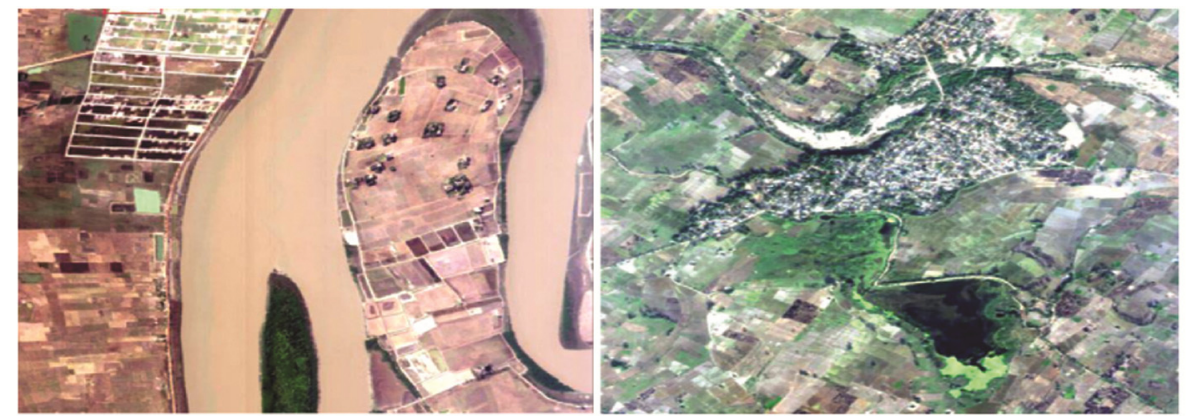

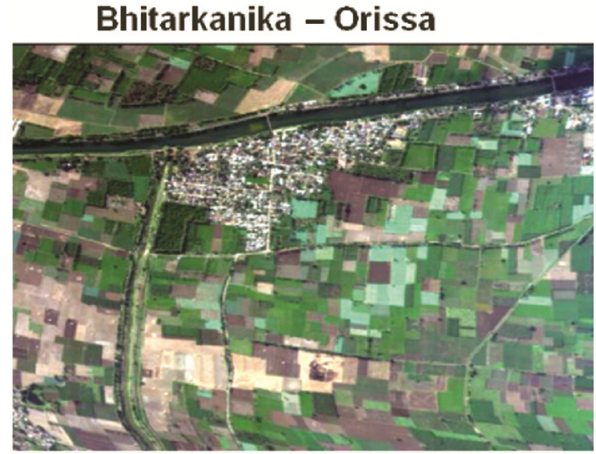

Vuyyur - Andhra Pradesh

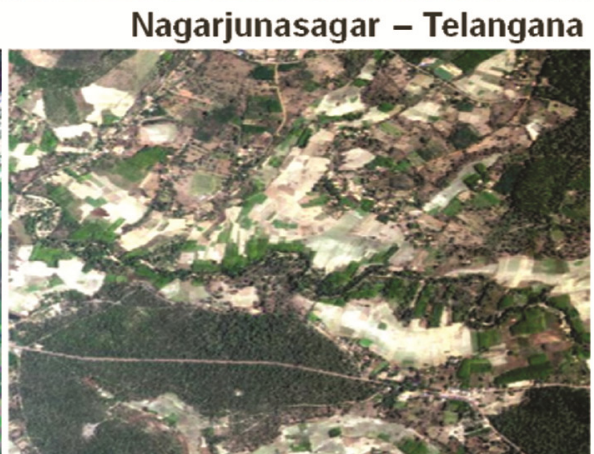

Shimoga forest - Karnataka

Figure 5. Examples of NCC view from AVIRIS-NG over different thematic targets. All AVIRIS-NG measurements are orthorectified.

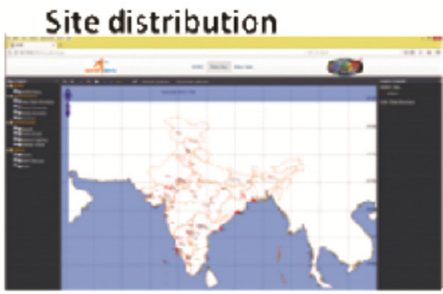

Base image of LISS 4 for each site

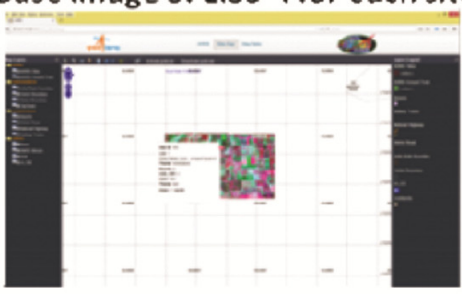

AVIRIS data download

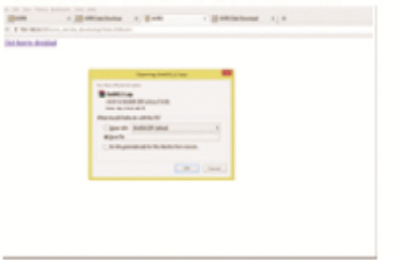

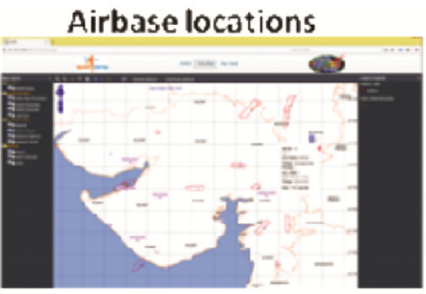

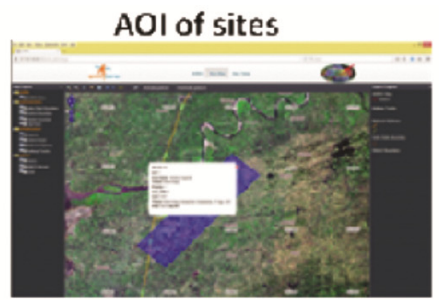

Site details

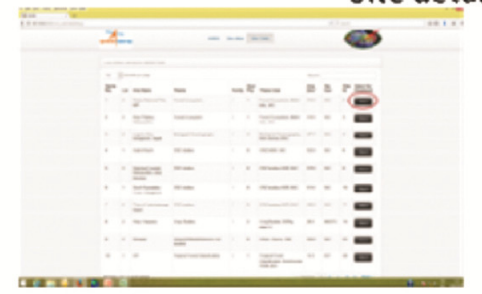

Ground truth information
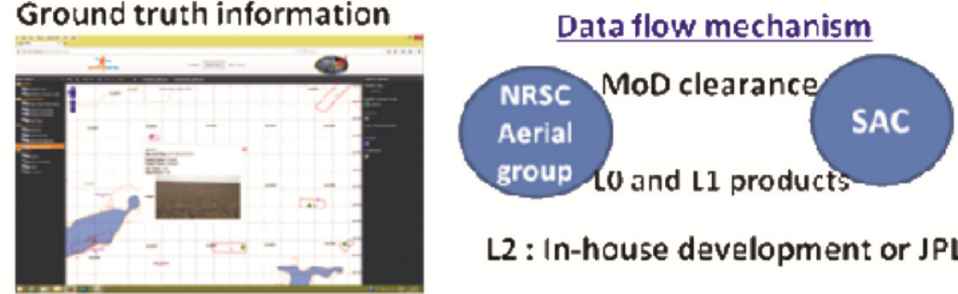

L2: In-house development or JPL

Figure 6. AVIRIS-NG data archival facility and data-flow mechanism.

environments in the post-monsoon condition. An AO programme for the Phase-2 campaign was initiated in February-May 2018 and the second part for Phase-2 is planned for the post-monsoon timeframe of 2019.
The experience gained using this AVIRIS-NG airborne campaign and utilization of spectroscopic measurements for different science applications is essential to inform development of operational products and algorithms for 
future space imaging spectrometer missions. Recently, the US Decadal Survey was released calling for this type of observation of the Earth as a high priority ${ }^{10}$. These space-based missions can provide such types of measurements for all of India in a timely manner to deliver fully operational applications. The end-use applications of thematic study would also support important key societal benefit areas such as: food security, forest health management and carbon sequestration, mineral exploration, water and air quality assessment, urban development and planning, cryospherere applications related to climate change and disaster-related applications.

1. Bhattacharya, B. K. and Chattopadhyay, C., A multi-stage tracking for mustard rot disease combining surface meteorology and satellite remote sensing. Comput. Electron. Agric., 2013, 90, 3544.

2. Bhattacharya, S., Majumdar, T. J., Rajawat, A. S., Panigrahy, M. K. and Das, P. R., Utilization of Hyperion data over Dongargarh, India, for mapping altered/weathered and clay minerals along with field spectral measurements. Int. J. Remote Sensing, 2012, 33(17), $5438-5450$.

3. Ramakrishnan, D. and Bharti, R., Hyperspectral remote sensing and geological applications. Curr. Sci., 2015, 108(5), 879-891.

4. Sahoo, R. N., Ray, S. S. and Manjunath, K. R., Hyperspectral remote sensing of agriculture. Curr. Sci., 2015, 108(5), 848-859.

5. Das, B. S., Sarathjith, M. C., Santra, P., Sahoo, R. N., Srivastava, R., Routray, A. and Ray, S. S., Hyperspectral remote sensing: opportunities, status and challenges for rapid soil assessment in India. Curr. Sci., 2015, 108(5), 860-868.

6. Ramakrishnan, D. and Sahoo, R. N., Network Programme on Imaging Spectroscopy and Applications (NISA): science plan and implementation strategy. Department of Science and Technology, Government of India, 2016.

7. Ajay Kumar, K., Thap, N. A. and Kuriakose, S. A., Advances in spaceborne hyperspectral imaging systems. Curr. Sci., 2015, 108(5), 826-832.

8. Green, R. O. et al., Imaging spectroscopy and the airborne visible/ infrared imaging spectrometer (AVIRIS). Remote Sensing Environ., 1998, 65(3), 227-248.

9. Green, R. O. et al., The Moon Mineralogy Mapper (M3) imaging spectrometer for lunar science: instrument description, calibration, on-orbit measurements, science data calibration and on-orbit validation. J. Geophys. Res.: Planets, 2012, 116(E10).

10. Anonymous, Thriving on Our Changing Planet: A Decadal Strategy for Earth Observation from Space, 2017-2027 Decadal Survey for Earth science and applications from space. The National Academies of Science, Engineering and Medicine (ISBN 978-0-30946757-5). The National Academies Press, Washington, DC, USA, 2017; doi:10.17226/24938.

ACKNOWLEDGEMENTS. We acknowledge the contributions of the ISRO and NASA teams, and the sponsors that made this campaign possible. A portion of this work was carried out at the Jet Propulsion Laboratory/California Institute of Technology, Pasadena, California, USA under contract with the National Aeronautics and Space Administration, USA.

doi: $10.18520 / \mathrm{cs} / \mathrm{v} 116 / \mathrm{i} 7 / 1082-1088$ 